\title{
- Métodos para avaliação da mineralização óssea de equiiinos em crescimento
}

\section{- Methods for evaluation of bone mineralization in foals}

\author{
Claudia Ehlers Kerber - CRMV-SP n 4285
}

Mestranda do Instituto de Ciências Biomédicas da Universidade de São Paulo



\section{RESUMO}

Algumas das principais doenças em eqüinos estão relacionadas aos membros locomotores, as quais podem advir de traumas ou distúrbios ósseos principalmente durante a fase de crescimento. No Estado de São Paulo, o desenvolvimento de pastagens com características tropicais, as alterações sazonais e a tendência à grande concentração de animais em pequenas propriedades resultam, freqüentemente, no aparecimento de distrofias ósseas, muitas vezes subclínicas, que depreciam o valor econômico dos eqüinos e limitam a sua capacidade de trabalho. O diagnóstico precoce dos desequilíbrios minerais assume grande importância na medida em que os distúrbios ósseos podem ser reversíveis se a causa for corrigida rapidamente. $\mathrm{O}$ autor faz uma revisão da fisiologia, da patofisiologia do osso, dos métodos de diagnóstico disponíveis e sugere o uso da dosagem da excreção fracional de fósforo como uma técnica de boa sensibilidade, precocidade, baixo custo e aplicabilidade para avaliar o nível de reabsorção do cálcio e do fósforo do tecido ósseo.

Unitermos : Cálcio, Fósforo, Clearance, Osso, Eqüinos

\section{Introdução}

As principais doenças dos cavalos estão ligadas aos membros locomotores (Monfort 1967, McIlwraith 1996 ), as quais podem advir de traumas ou de distúrbios ósseos, observados principalmente durante a fase de crescimento.

No Estado de São Paulo, o desenvolvimento das pastagens de características tropicais, as alterações sazonais e a tendência à grande concentração de animais devido ao alto custo da terra freqüentemente resultam em uma alimentação deficiente em minerais e aparecimento de distrofias ósseas, que depreciam o valor econômico dos eqüinos e muitas vezes os inutilizam para o trabalho (Haddad 1987, Cunha 1991).
O diagnóstico precoce dos desequilíbrios minerais assume grande importância na medida em que os distúrbios ósseos podem ser reversíveis se a causa for corrigida rapidamente. O método de diagnóstico deve ser sensível, precoce e aplicável em larga escala. Um método bastante recomendado pela sua sensibilidade e precocidade é o clearance fracional de fósforo (Joyce 1971 et al., Coffman 1981, Traver 1977, Balarin 1990 ). Mais recentemente a dosagem de paratormônio pelo método de radioimunoensaio (Allen et al. 1987, Enbergs et al. 1996) e de imunoradiometria de hormônio intacto (Warren 1991) foram validados para uso em eqüinos, mas ainda não há estudos publicados com o seu uso em larga escala. Finalmente, ainda está em fase de avaliação o uso 
de marcadores ósseos como método de diagnóstico de osteopatias (Harris; Gray 1997 ).

\section{Considerações sobre o cálcio e o fósforo do ponto de vista nutricional em eqüinos}

Os cavalos são altamente suscetíveis a sofrer com dietas contendo níveis inadequados de cálcio e/ ou fósforo, mais do que qualquer outro mineral (Lewis 1982).

Segundo a recomendação do National Research Council (1989) e de diversos autores, a quantidade de ração concentrada oferecida aos eqüinos nunca deveria ultrapassar $50 \%$ do total da dieta. A outra metade deve se constituir de volumoso, mais freqüentemente administrado na forma de pastejo. Os concentrados são ricos em energia e de maneira geral os grãos são ricos em fósforo e pobres em cálcio. Segundo Lewis (1982), o excesso de ingestão de concentrado é a principal causa de aparecimento de lesões ósseas nos cavalos.

As espécies vegetais mais recomendadas pelos agrônomos para formar pastos para eqüinos no Estado São Paulo são as de crescimento estolonífero, que se recuperam bem após o pastejo contínuo (Corsi 1984) e se adaptam bem ao clima tropical e ao hábito de pastejo rasteiro dos eqüinos (Haddad 1987 ).

O Coast Cross é o mais popular, constituindose num híbrido do capim Cynidom sp desenvolvido na Georgia. É resistente ao frio e permanece verde o ano inteiro, mas é exigente quanto à fertilidade e responde bem à adubação (Haddad 1987 ). Recentemente o Tifton tem sido introduzido. Suas propriedades nutritivas e de crescimento são semelhantes às do Coast Cross.

Para que a pastagem tenha uma maior capacidade de suporte (número de animais por área), é necessário que se adote um bom manejo, seja com uma alta produção que suporte um maior número de animais, ou com uma taxa de lotação ajustada ao seu nível de produção. Pode-se conseguir uma maior produção com uma boa adubação, baseada em análise de solo e adotando-se piquetes menores com rotação, respeitando-se o ciclo de crescimento da planta. As pastagens que não são submetidas à adubação sob uso constante, além de não suportarem um grande número de animais, produzem plantas de pouco valor nutritivo, obrigando ao consumo de concentrados ou feno como forma de complementar a dieta e manter o desenvolvimento dos animais.
Ainda que se adotem os cuidados necessários para uma máxima produção, as pastagens tropicais apresentam cerca de $80 \%$ da produção total no período denominado "verão agrostológico" (setembro a abril) e somente $20 \%$ no inverno. O seu valor nutritivo também diminui no inverno. Os fatores limitantes do crescimento são a temperatura e o fotoperíodo, além da diminuição pluviométrica. Assim, enquanto uma pastagem de 1 hectare bem manejada no verão suporta facilmente 5 cavalos, no inverno pode não ser suficiente nem mesmo para dois deles (Lewis, 1982 ).

As rações comercialmente disponíveis para cavalos têm o milho como principal fonte de energia e a base da alimentação dos eqüinos no nosso meio é com gramíneas. Nem os grãos nem as gramíneas contém suficiente quantidade de cálcio para suprir as necessidades de um cavalo em crescimento (Cunha 1991), especialmente se forem de má qualidade ou estiverem muito maduros. Desta forma, as necessidades diárias dos cavalos com relação ao fósforo são satisfeitas ou estão em excesso, enquanto que as de cálcio estão bem aquém do necessário, devendo ser corrigidas através de suplementação mineral e/ou fornecendo forragem à base de leguminosas. As leguminosas (alfafa, trevo) possuem 3 a 6 vezes mais cálcio do que as gramíneas e a mesma quantidade de fósforo, mas em regiões tropicais o consorciamento de gramíneas/leguminosas não é recomendado (Haddad 1987), de forma que os criadores são obrigados a administrar feno de leguminosas (alfafa) se pretendem utilizar este alimento.

De uma forma geral o balanceamento das rações para equiinos é feito com base na idade/peso e tipo de trabalho que eles desenvolvem não levando em conta estas variações sazonais ou a qualidade da forragem oferecida, embora isto seja da maior importância, já que respeitando-se a proporção de fornecer $50 \%$ da ração em concentrado, um cavalo em crescimento ingere aproximadamente 2 a 2,5\% do seu peso vivo em forragens por dia (National Research Council ).

\section{O esqueleto eqüino, fisiologia do crescimento e patofisiologia}

\section{Desenvolvimento normal dos ossos longos}

Os ossos longos se desenvolvem a partir da cartilagem por um processo de ossificação endocondral. No feto, a matriz dos ossos é composta somente de cartilagem. Os centros de ossificação se desenvolvem no centro dos futuros ossos longos (diáfise) e 


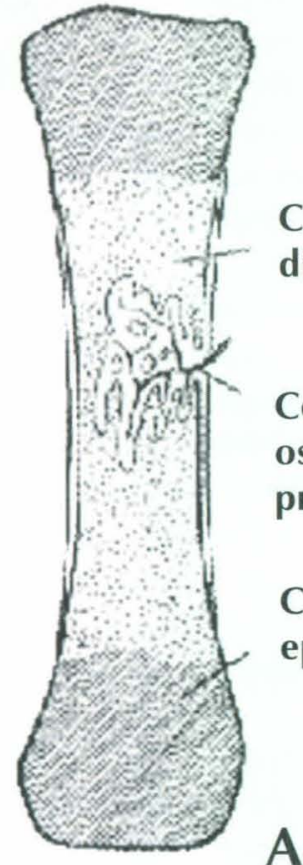

Cartilagem diafisária

Centro de ossificação primário

Cartilagem epifisiária

A

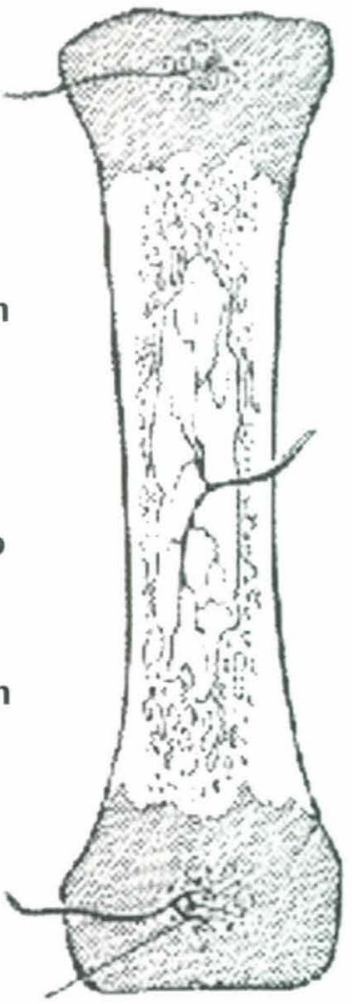

Centro de

B ossificação secundário

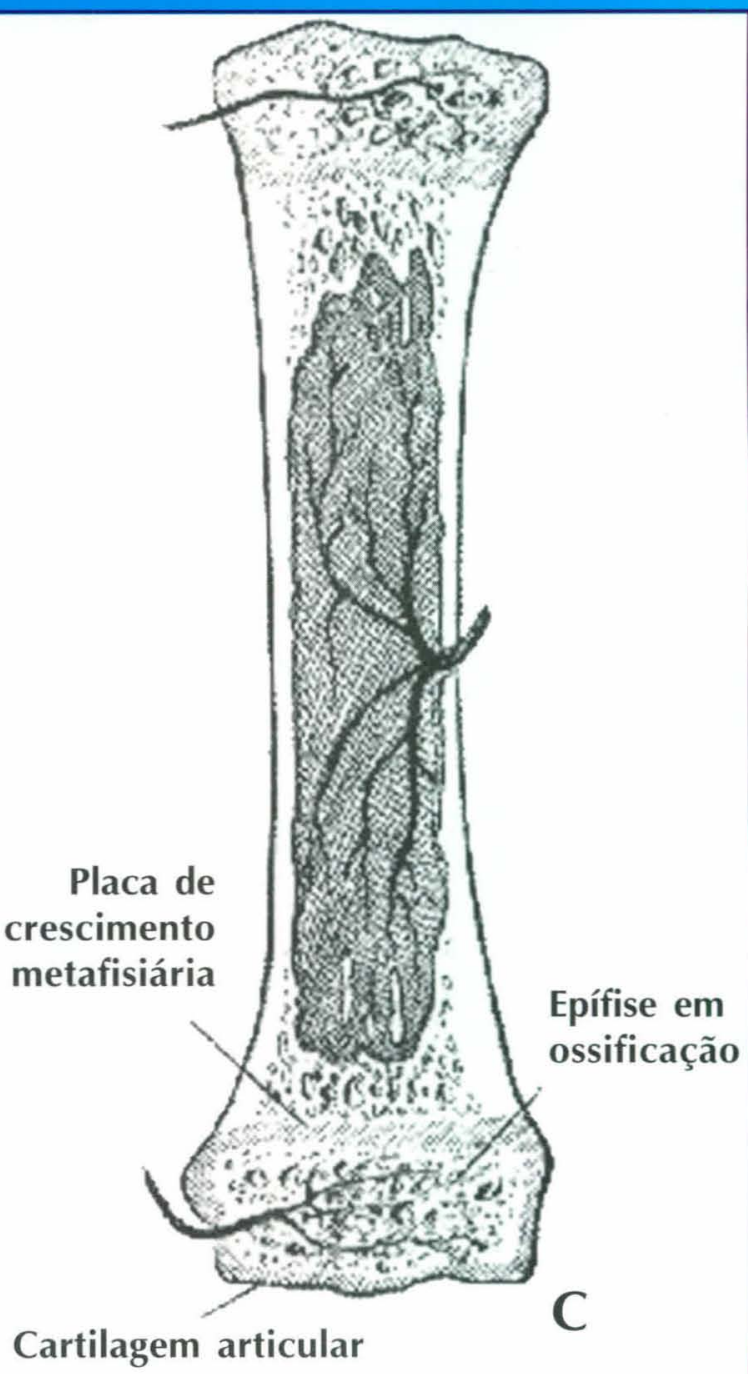

Fonte: World Equine Veterinary Review

também nas extremidades (epífise ). Com o início da ossificação, uma epífise óssea se desenvolve a cada extremidade e uma diáfise óssea se desenvolve no centro.

Entre estes dois centros de ossificação está a placa de crescimento metafisário (também chamada fise), que permite o alongamento do osso que acompanha o crescimento após o nascimento. No momento oportuno, ocorre a ossificação da fise. O fechamento da fise de cada osso se dá em momentos diferentes; como regra geral pode-se dizer que os ossos distais fecham antes dos ossos proximais.

\section{Mecanismos fisiológicos da homeostase de cálcio e fósforo}

A manutenção da homeostasia do cálcio e do fos- fato depende principalmente do trato intestinal, do esqueleto e dos rins. Além disto uma contribuição essencial é dada pela pele e fígado.

O íon cálcio é de fundamental importância para todos os sistemas biológicos e a sua concentração deve se situar entre limites estreitos de tolerância fisiológica entre os diversos compartimentos. O íon fosfato também é de importância crítica em todos os sistemas biológicos.

O nível normal de cálcio no plasma de eqüinos adultos é de 10,2 a 14,3 mg/dl (Coffman 1981, Schryver et al. 1971) e se mantém aproximadamente nestes níveis durante toda a vida do animal. $O$ de fosfato inorgânico é de 2,1 a $5,9 \mathrm{mg} / \mathrm{dl}$, no entanto, da mesma forma como acontece em humanos (Portale 1990 ), a fosfatemia em cavalos também varia com a idade (Bauer 1990, Enbergs et al. 1996 ). 


\section{O papel do intestino}

Vários fatores influenciam a absorção do cálcio e do fósforo. Normalmente cerca de $50 \%$ do cálcio ingerido é absorvido, mas a eficiência da absorção nos eqüinos diminui na sobrecarga e aumenta quando os níveis são deficientes na dieta (Schryver et al. 1971 ). A eficiência da absorção intestinal aumenta ainda durante períodos de maior intensidade na mineralização óssea, como crescimento e prenhez e também na lactação. Com relação à absorção de fosfato nos eqüinos, a porcentagem absorvida na dieta é relativamente constante e depende principalmente da fonte. Basicamente os fosfatos solúveis são mais facilmente absorvidos e podemos estimar a absorção de fósforo em 29-32\% nos concentrados, 44-46\% nas forragens e como média geral, 58\% nos melhores suplementos minerais (Lewis 1982 ).

Fitatos e oxalatos se ligam aos cátions, diminuindo a sua absorção. As gramíneas de pastagens tropicais e subtropicais podem apresentar alto nível de oxalato, diminuindo a absorção de cálcio e podendo causar o hiperparatireoidismo secundário nutricional (Walthall; McKenzie 1976 ). Os grãos de cereais são ricos em fitatos e o seu conteúdo aumenta com o amadurecimento.

\section{O papel do rim}

O rim filtra diariamente uma grande quantidade de cálcio não ligado a proteínas, mas cerca de $98 \%$ é reabsorvido. Reabsorve também 80 a $97 \%$ do fósforo filtrado. Quando o nível plasmático aumenta, aumenta a filtração e também a reabsorção; no entanto, os mecanismos de reabsorção são rapidamente saturados e a excreção, então, aumenta em proporção ao fosfato filtrado.

\section{O papel do esqueleto}

Tanto a formação e mineralização do osso como a reabsorção dependem da concentração plasmática de cálcio e fósforo. Por sua vez, o esqueleto é o grande reservatório de cálcio e de fósforo disponível para a manutenção dos níveis normais destes minerais no sangue.

\section{O papel dos hormônios}

Os principais hormônios controladores da calcemia são o paratormônio, a calcitonina e a vitamina D, embora outros como os corticóides da adrenal, estrógenos, tiroxina, somatotropina, glucagon, possam também contribuir na homeostase do cálcio.
A ação mais evidente do paratormônio é mobilizar o cálcio das reservas do esqueleto e jogá-lo no fluído extracelular, aumentando a concentração do cálcio plasmático. Ele também aumenta a reabsorção do cálcio no rim e aumenta a excreção urinária de fósforo.

O único estímulo necessário para a liberação do paratormônio é a variação na calcemia.

A calcitonina é produzida pelas células $\mathrm{C}$ da tireóide. O estímulo para a sua liberação é o aumento da concentração do cálcio iônico no sangue. A ação da calcitonina é antagônica à do paratormônio com relação à reabsorção óssea.

O terceiro importante hormônio envolvido na regulação do metabolismo do cálcio e remodelação do esqueleto é o colecalciferol (vitamina $\mathrm{D}_{3}$ ), que pode ser absorvido pelo intestino ou sintetizado na epiderme através de reação catalisada pela radiação ultravioleta do sol. Em regiões tropicais a insolação abundante garante excelente aporte de vitamina $\mathrm{D}_{3}$ (Capen 1980 ).

\section{Patofisiologia}

Em cavalos, o mais freqüente desequilíbrio nutricional ligado aos minerais é a ingestão excessiva de fósforo ou insuficiente de cálcio (Capen 1993). A ingestão excessiva de fósforo resulta numa absorção intestinal excessiva e hiperfosfatemia. A hiperfosfatemia estimula a paratireóide indiretamente, levando à reabsorção óssea e aumento da fosfatúria. Da mesma forma, a ingestão insuficiente de cálcio determina uma hipocalcemia, que leva à estimulação da paratireóide.

A ingestão contínua de uma dieta desbalanceada leva a um estado permanente de hiperparatireoidismo compensatório e ao desenvolvimento progressivo de distúrbios metabólicos do osso por reabsorção da matriz óssea, decorrente da ação do paratormônio (Schryver et al. 1974, 1975 ).

Os cavalos com este tipo de distúrbios geralmente se alimentam de dietas ricas em grãos e com forragens de baixa qualidade. Este tipo de dieta normalmente é palatável e nutritiva, exceto pelo desequilíbrio cálcio/fósforo, de tal forma que o animal se desenvolve com peso e tamanho normais mas podendo apresentar fragilidade óssea clínica ou subclínica.

Quando as lesões se tornam evidentes clinicamente, algumas das osteopatias mais freqüentemente observadas são as seguintes:

- Osteocondrose - É um defeito da ossificação osteocondral que resulta em diversas manifestações, 


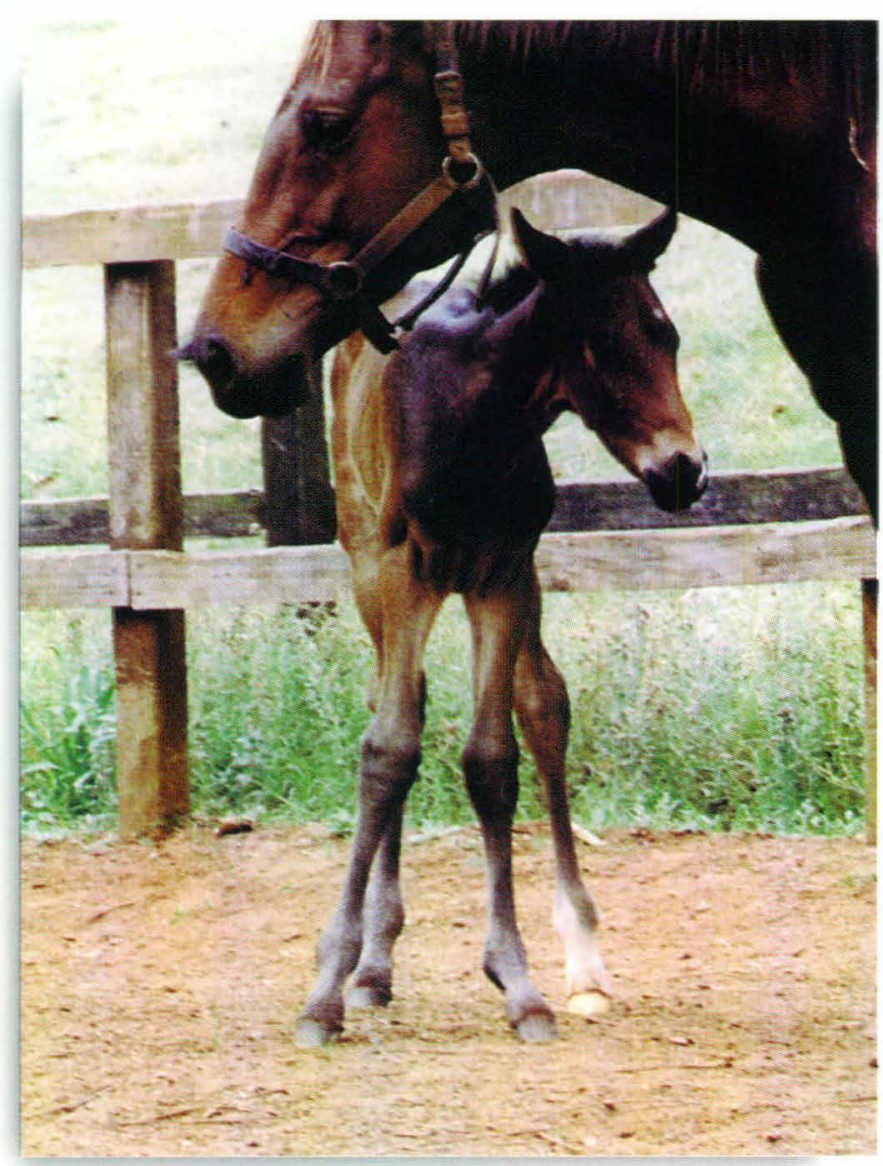

Figura 1 - Deformidade angular em potros

dependendo do local aonde ela se instala. Ela pode surgir na forma de lesões císticas no osso ou como defeito na cartilagem articular. Freqüentemente estas lesões são dolorosas e muitas vezes somente diagnosticadas por exame radiológico ou quando há manifestação de dor, mormente na intensificação do treinamento. O desequilíbrio nutricional, especialmente relacionado aos macrominerais, é preponderante no aparecimento destas lesões.

- Deformidades angulares ou flexurais (figuras 1 e 2) - Quando associada ao desenvolvimento. Uma das causas pode ser por desequilíbrio mineral .

- Epifisite - Quando a cartilagem de crescimento não é substituída por osso e os vasos não conseguem penetrar na zona de crescimento, o osso não se organiza, há necrose nesta área e podemos observar clinicamente o alargamento no local da placa de crescimento distal do osso rádio ou do metacarpo e metatarso distal em potros de até dois anos. O local se mostra inflamado e em casos mais graves pode haver manifestação dolorosa, acom-

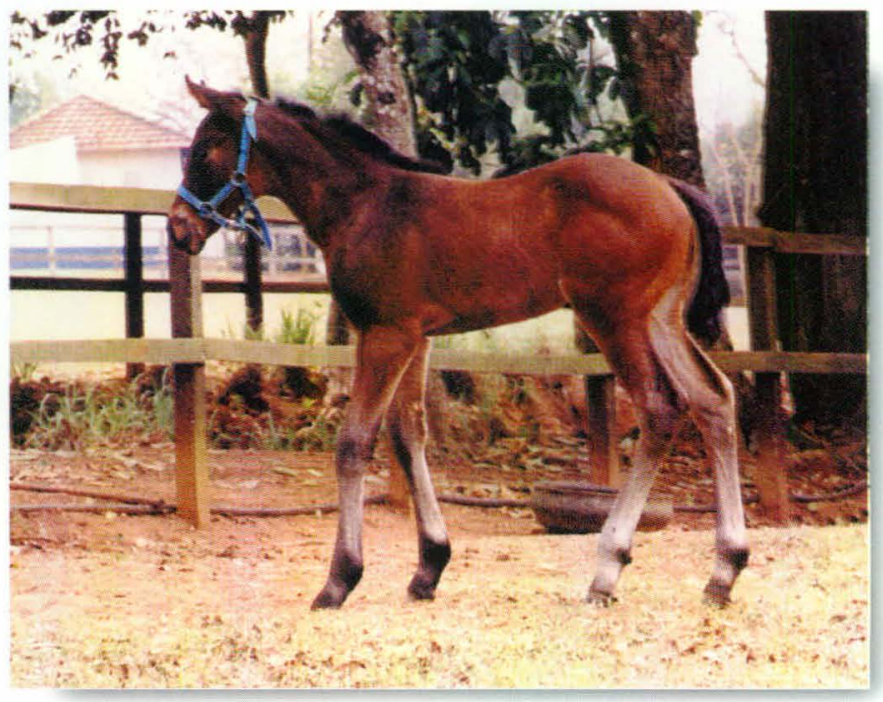

Figura 2 - Deformidade flexural em potros

panhada de manqueiras. Também são comuns as sinovites, especialmente no tarso e no carpo (figura 3). O que se observa rotineiramente são manifestações leves deste processo. Estima-se que

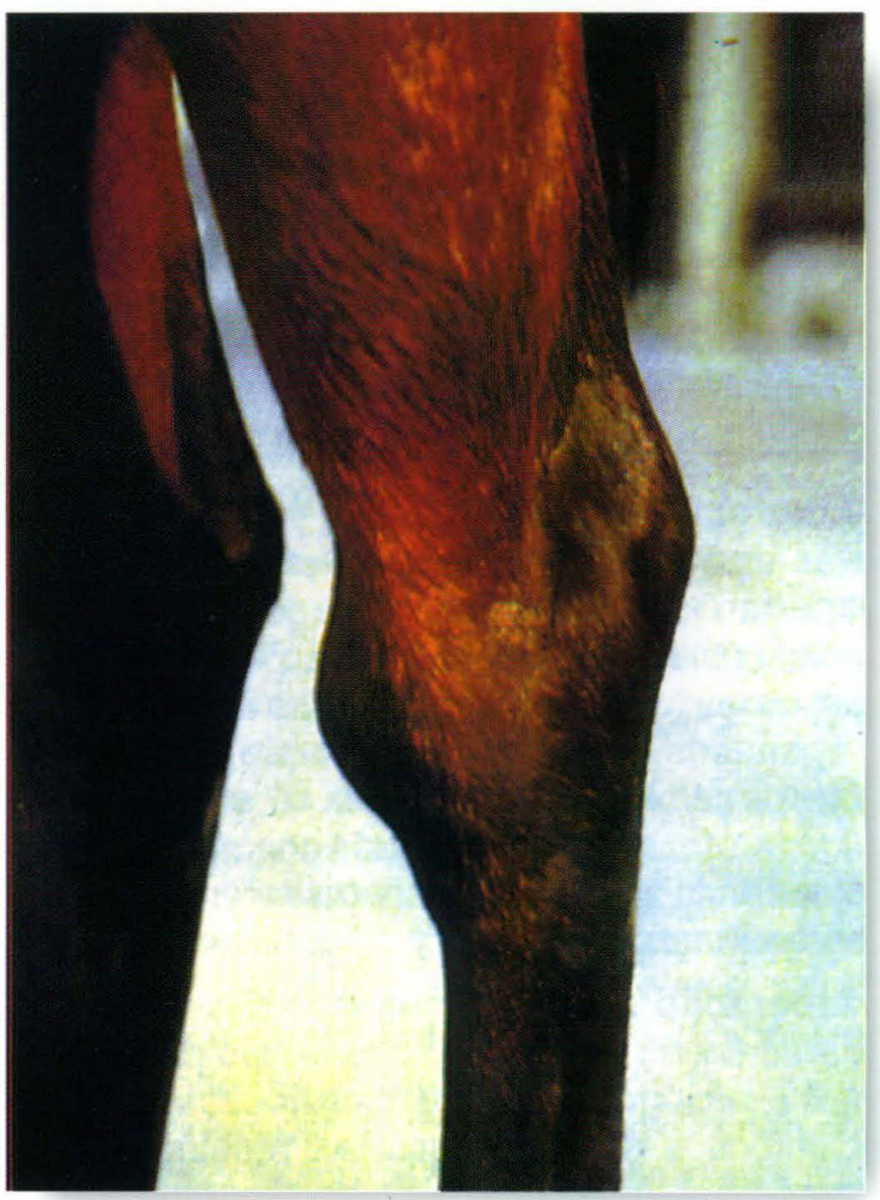

Figura 3 - Sinovite no tarso 


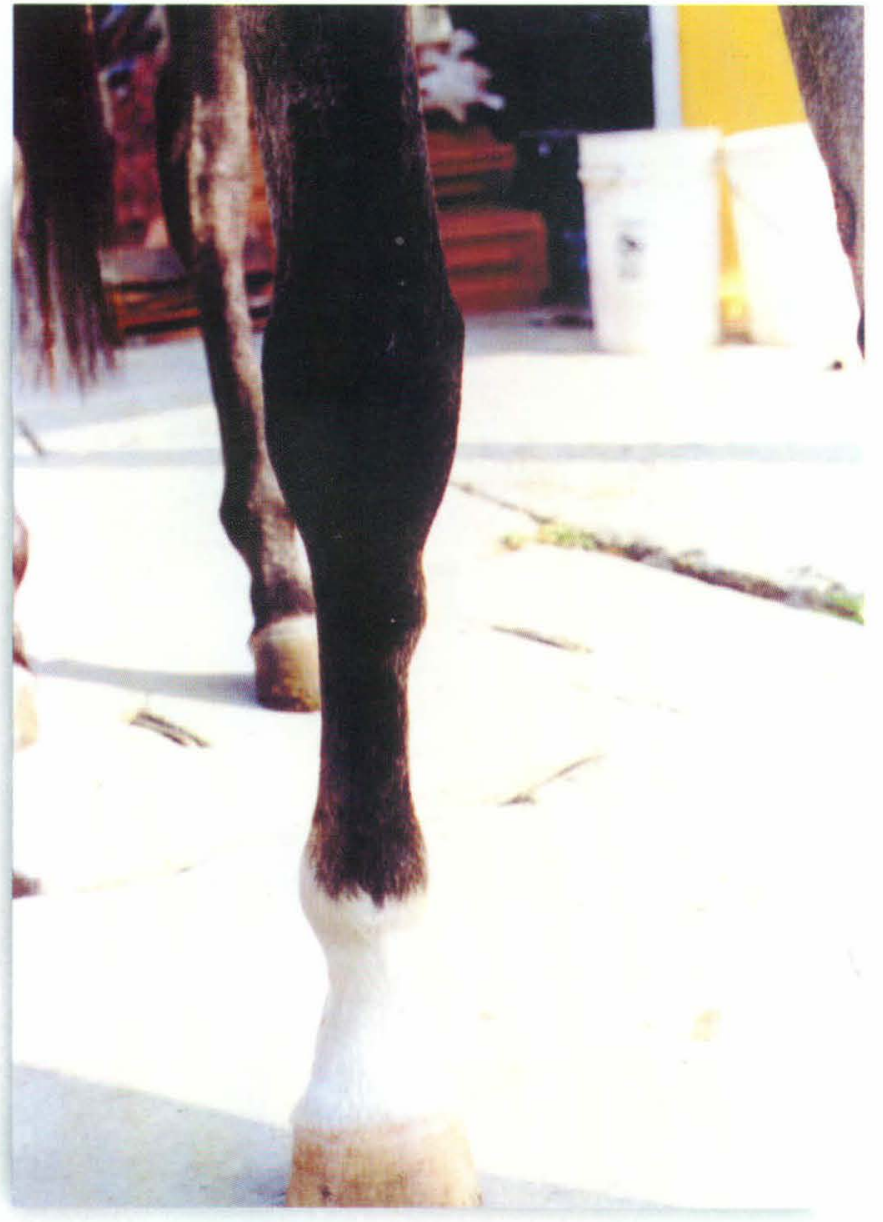

Figura 4 - Exostose em animal em treinamento

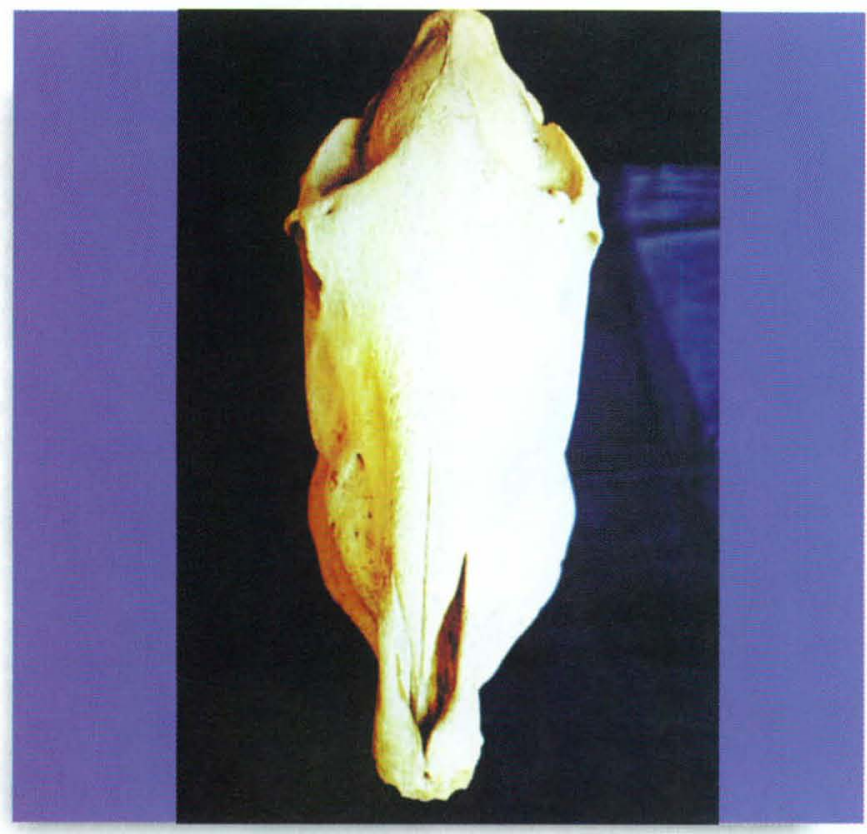

Figura 5 - Crânio de animal portador de Osteodistrofia Fibrosa. Notese $o$ alargamento dos ossos da face. Fotografia cedida pelo Dr. Romeu Macruz
$73 \%$ a $88 \%$ dos problemas de crescimento em potros são devido a deformidades de angulação ou epifisite.

- Distúrbios ósseos no cavalo em treinamento O cavalo em treinamento está sujeito a muitos tipos de lesão, como fraturas ósseas, inflamação do periósteo, microfraturas, exostoses (figura 4 ), etc. Muitas destas patologias podem ter sua origem no desenvolvimento inadequado do osso. O papel dos macrominerais é muito importante e altos níveis de fósforo na dieta produzem lesões ósseas em potros jovens sem sinais clínicos de hiperparatireoidismo (Schryver et al. 1971). Altos níveis de cálcio (3 vezes a recomendação do NRC) não produziram lesões ósseas (Jordan et al. 1975).

- Osteodistrofia fibrosa - Em animais adultos com estágio avançado da doença, podemos observar lesões severas, especialmente o alargamento dos ossos da mandíbula e maxila, distúrbio conhecido como "cara inchada" (figuras 5 e 6 ), e manqueira evidente. Estes sinais aparecem em decorrência do aumento da atividade osteoclástica das lamelas ósseas externas com ruptura das inserções dos tendões e das trabéculas ósseas que dão suporte à cartilagem articular. Nos ossos da face há uma deposição em excesso de tecido conjuntivo e osteóide por estímulo dos osteoblastos, daí o alargamento progressivo dos ossos.

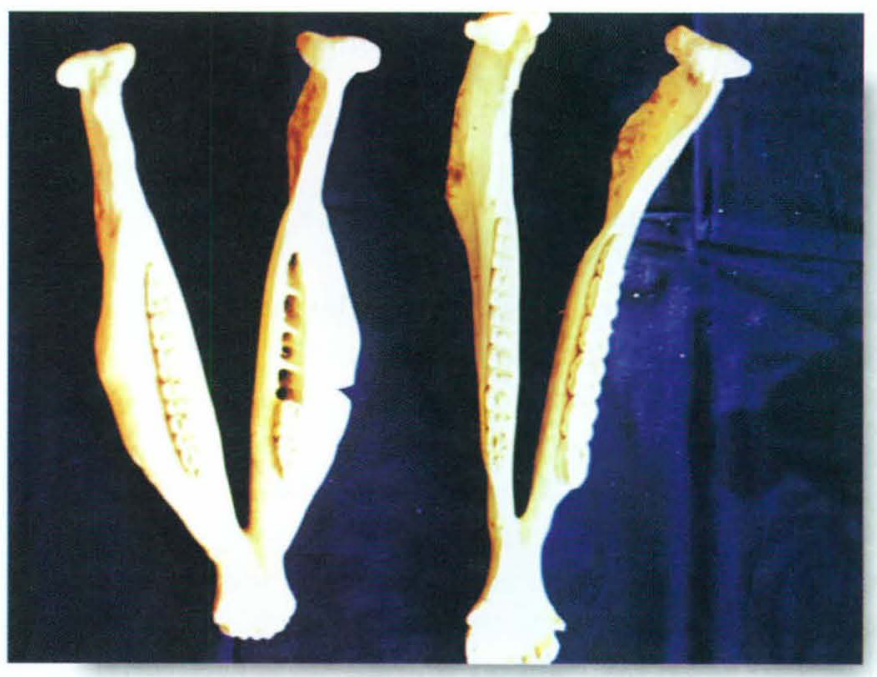

Figura 6 - Fotografia comparativa da mandíbula de um animal normal (à direita) com a de um animal com Osteodistrofia Fibrosa (à esquerda). Observe-se o alargamento do osso e a perda dos dentes. Fotografia cedida pelo Dr. Romeu Macruz 


\section{Considerações sobre o diagnóstico}

O diagnóstico precoce dos desequilíbrios minerais assume grande importância na medida em que os distúrbios ósseos podem ser reversíveis, se a causa for corrigida rapidamente. O método de diagnóstico deve ser sensível, precoce e aplicável em larga escala. A anamnese e o exame clínico são imprecisos, já que a fragilidade óssea pode não se manifestar clinicamente até que o esqueleto esteja comprometido irreversivelmente (Adams 1987, Savaje et al. 1992, McIlwraith 1993, 1996, 1997). Diversos autores já demonstraram as limitações das dosagens sangüíneas de cálcio, fósforo e fosfatase alcalina (Argenzio et al. 1974, Coffman et al. 1978, 1981, Capen et al. 1983 ) bem como da análise da ração (Caple et al. 1982 a,b, Lewis 1982).

Um método bastante recomendado pela sua sensibilidade, custo e precocidade é o clearance fracional de fósforo (Joyce et al. 1971, Coffman 1981, Traver 1977, Lewis 1982, Balarin 1990 ). Mais recentemente a dosagem de paratormônio pelo método de radioimunoensaio (Allen et al. 1987, Enbergs et al. 1996) e de imunoradiometria de hormônio intacto (Warren 1991) foram validados para uso em eqüinos, mas ainda não há estudos publicados com o seu uso em larga escala. Finalmente, ainda está em fase de avaliação o uso de marcadores ósseos como método de diagnóstico de osteopatias (Harris; Gray 1997 ).

\section{Clearance fracional de fósforo}

Como descrito acima, os desequilíbrios minerais mais observados no nosso meio são a carência absoluta de cálcio ou a sua deficiência relativa por excesso de ingestão de fósforo. Isto leva a um aumento na atividade do paratormônio, promovendo maior reabsorção óssea e aumento na excreção renal de fósforo. Desta forma, o diagnóstico do aumento do clearance fracional do fósforo pode indicar a ocorrência excessiva de reabsorção e enfraquecimento do esqueleto.

Para determinar a perda renal de um eletrólito, classicamente seria necessário uma coleta de urina durante 24 horas, por causa da variação na quantidade de água da urina. Neste caso, a concentração do eletrólito multiplicado pelo volume urinário daria a excreção urinária diária, no entanto, a coleta de urina durante 24 horas é muito difícil na clínica de eqüinos. Uma alternativa seria comparar o clearance do fósforo com o clearance da creatinina endógena e calcular o clearance fracional, eliminando o volume dos cálcu- los. A creatinina é produzida continuamente pelo metabolismo muscular e aproximadamente $90 \%$ dela é excretada quando comparada com clearance da inulina (Knudsen 1959 ), refletindo, assim, o ritmo de filtração glomerular.

O clearance renal de uma determinada substância é representado pela concentração do eletrólito na urina, dividida pela concentração no soro, multiplicado pelo fluxo urinário. Por definição ele representa o volume de plasma que a cada minuto fornecerá a quantidade da substância presente na urina $(\mathrm{ml} / \mathrm{min})$.

$\mathrm{O}$ procedimento para o cálculo do clearance se inicia com a coleta de urina e sangue sem anticoagulante, os quais devem ser mantidos refrigerados. O soro é separado por centrifugação e realiza-se a dosagem de fósforo por método colorimétrico e de creatinina pelo método do picrato alcalino de Jaffe nas amostras de sangue e de urina. Os valores encontrados são colocados na fórmula para cálculo da excreção fracional ou clearance fracional :

\section{Clearance fracional ou excreção fracional:}

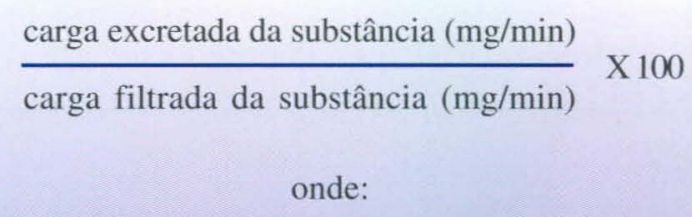

Pi: Fósforo inorgânico

Carga excretada de Pi :

Fluxo urinário ( $\mathrm{ml} / \mathrm{min}$ ) X Conc.Pi na urina $(\mathrm{mg} / \mathrm{ml})$

Carga filtrada de $\mathbf{P i}$ :

Ritmo de filtração glomerular ( $\mathrm{ml} / \mathrm{min}$ )

$\mathrm{X}$

Conc.Pi filtrável no plasma (mg/ml)

A qual pode ser expressa da seguinte maneira:

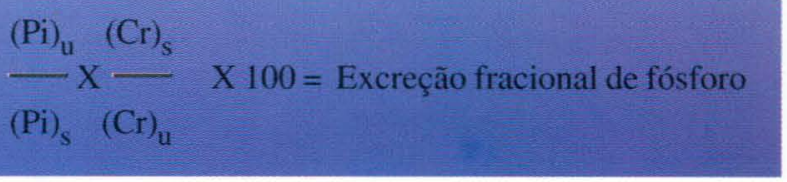

Os valores normais de clearance fracional se mostram dentro de limites bem precisos em cavalos sãos recebendo dieta balanceada e se situam entre 0 e $0,5 \%$ (Coffman 1978). Os valores aumentam bastante quando 
KERBER, CLAUDIA EHLERS. Métodos para avaliação da mineralização óssea de eqüinos em crescimento. Revista de Educação Continuada do CRMV-SP / Continuous Education Journal CRMV-SP. São Paulo, volume 2, fascículo 1, p. 17 - 25, 1999.

os animais são submetidos a dietas ricas em fósforo ou pobres em cálcio. Desta maneira, valores acima de 0,5 são indicativos de excreção renal excessiva de fósforo em decorrência do processo de hiperparatireoidismo compensatório.

Os valores aumentados têm grande importância nos animais em crescimento e deve-se proceder a uma análise cuidadosa da ração administrada e ao seu balanceamento. Após implantação da dieta corrigida podemos observar um retorno progressivo aos níveis nor- mais de excreção de fósforo em aproximadamente 30 dias.

Em animais estabulados, os valores de clearance costumam se situar entre 0,5 e 2,5 , mas podem aumentar em até $42 \%$ em cavalos estabulados clinicamente normais (Caple et al. 1982 ).

Assim, o clearance fracional de fósforo é uma técnica útil e aplicável em larga escala, indicando desequilíbrio dos macrominerais cálcio e fósforo na dieta e enfraquecimento do esqueleto.

\section{SUMMARY}

Some of the main equine diseases are related to the musculoskeletal system, mainly during the bone developing in young animals. In São Paulo state, Brazil, the use of tropical grasses for grazing, the seasons and a general tendency to concentrate a large number of horses in small areas, frequently results on bone diseases, sometimes subtle, which depreciate the economic value of the horses and decrease the chance of a maximum performance. An early diagnosis of mineral imbalance play an important role once the bone diseases can be reversible when corrected on time. The author presents a review of physiology and pathophysiology of the bone. Also describe the methods available for diagnostic and suggest the phosphorus clearance as a sensitive, early, low cost and easy way to evaluate calcium and phosphorus mobilisation from the bone.

Uniterms : Calcium, Phosphorus, Clearance, Bone, Equine

\section{BIBLIOGRAFIA DE APOIO}

1. ADAMS, O.R. : Lameness in horses, 4. ed. Philadelphia: Lea \& Febiger, 1987.

2. ALLEN,J.R.;LIN,Y.C.,STRAIT,J.R.,MODRANSKY,P.D.: Radioimmunoassay for parathyroid hormone in equids, American Journal Veterinary Research, v.48,n.4 p.586-9, 1987.

3. NATIONAL RESEARCH COUNCIL. Nutrients requirements for horses 5. ed. Washington, National Academy Press, 1989.

4. ARGENZIO,R.A., LOWE,J.E.,HINTZ,H.F.,SCHRYVER,H.F.: Calcium and phosphorus homeostasis in horses. Journal of Nutrition, v.104, p.18-24, 1974.

5. BALARIN, M.R.S.: Avaliação do estado nutricional de cálcio e fósforo em bovinos por meio da análise bioquímica da urina. Botucatu, Br, 1990. Dissertação (Mestrado) - UNESP,

6. BAUER, J,E. Normal Blood Chemistry.In: KOTERBA, A.M.; DRUMMOND,W.H.;KOSCH,P.C. (ed) Equine clinical neonatology, Philadelphia, Lea\&Febiger, 1990, p.602-614.

7. CAPEN,C.C.: The calcium regulating hormones : parathyroid hormone, calcitonin and cholecalciferol In: MCDONALD, L.E. (ed): Veterinary Endocrinology and reproduction 3. ed. Philadelphia, Lea\&Febiger, 1980, p.60-130.

8. CAPEN,C.C.: Nutritional secondary hyperparathyroidism In: ROBINSON, N. E. ed. Current therapy in equine medicine, Philadelphia, W.B.Saunders, 1993, p.160-3.
9. CAPLE,I.W; BOURKE,J.M,;ELLIS,P.G.: An examination of the calcium and phosphorus nutrition of thoroughbred racehorses, Australian Veterinary Journal, v.58, p.132-135, 1982 a.

10. CAPLE,I.W.;DOAKE,P.A.;ELLIS,P.G.: Assessment of the calcium and phosphorus nutrition in horses by analysis of urine, Australian Veterinary Journal, v.58, p125-135, 1982 b.

11. COFFMAN,J.R.: Bone and muscle defects in foals. Modern Veterinary Practice, v.54, n.13, p53-54,1973

12. COFFMAN,J.R.: Clearance ratios in the horse. In: DENVER, VETERINARY MEDICAL ASSOCIATION SEMINAR. 1978.

13. COFFMAN,J.R.: Calcium and phosphorus physiology and pathophisiology. In: Equine clinical chemistry and pathophysiology. Veterinary Med.Publishing Company, Kansas, 1981

14. CORSI,M. : Effects of nitrogen ratios and harvesting intervals on dry matter production, tillering and quality of the tropical grasses Panicum maximum, 1984, PhD Thesis, Ohio State University, USA.

15. CUNHA, T.J.: Pasture for horses. In: Horses feeding and nutrition, California: Academic Press. 1991, p.274-93.

16. ENBERGS,H;KARP,H.P.;SCHONHERR,U. : Course of blood levels of calcium, inorganic phosphate, alkaline phosphata- 
se parathyroid hormone and calcidiol in one and two year old thoroughbred horses: Deutsche Tierarztl Wochenschr, v.103, n.12, p.491-493, 1996.

17. HADDAD,C.: Pastagens. In : CARVALHO, R.T.L., HADDAD, C. (ed) Pastagens e alimentação de eqüinos. Piracicaba: FEALQ, 1987.

18. HARRIS,P.;Gray,J.A.: Biochemical bone markers. In ROBINSON,N.E. (ed) Current therapy in equine medicine, Philadelphia: W.B.Saunders, 1997, p.112-115.

19. JORDAN, R.M.; MYERS, V.S.; YOHO, B.; SPURRELL, F.A.,Effect of calcium and phosphorus levels on growth, reproduction and bone development in ponies, Journal of Animal Science, v.40, p.I, 1975.

20. JOYCE,J.R.;PIERCE,K.R.,ROMANE,W.M.;BAKER,J.M.: Clinical study of nutritional secondary hyperparathyroidism in horses. Journal of American Veterinary Medical Association v.158, p2033-2042, 1971

21. KNUDSEN,E.: Renal clearance studies on the horse, inulin, endogenous creatinine and urea, Acta Vet Scand, v. 1, n. 1, p. 52, 1959.

22. LEWIS,L.D. (ed) Feeding and care of the horse, Philadelphia: Lea\&Febiger, 1982.

23. MCILLWRAITH,W.:What is developmental orthopedic disease, osteochondrosis, ostochondritis, metabolic bone disease In: AMERICAN ASSOCIATION FOR EQUINE PRACTITIONERS CONVENTION, 39. Proceedings Book, 1993.

24. MCILWRAITH,W.: The equine skeleton: How does bone grow and how do abnormalities in the SSZ \developmental process affect soundness I. World Equine Veterinary Review, v.1, n.2, p.25-9,1996.

25. MCILWRAITH,W.: The equine skeleton II. World Equine Veterinary Review, v. 12, n.1, p.22-7,1997.
26. MONFORT,T.N.: A radiografic survey of epiphyseal maturity in thoroughbred foals from birth to three years of age. Proceedings of the American Association for Equine Practitioners, p33-37, 1967

27. PORTALE,A.A. : Blood calcium, phosphorus and magnesium. In Favus,M.J. (ed): Primer on metabolic bone diseases and disorders of mineral metabolism. American Society for Bone and Mineral Research, New York, Lippincot-Raven Pub, 1990, p. 93-6.

28. SAVAJE,C.J.;MCCARTHY,R.N.;JEFFCOTT,L.B.Induction of dyschondroplasia in foals.II.Effects of dietary phosphorus and calcium. In: Jeffcott,L.B., Dalin, G.eds. Equine Osteochondrosis in the 90's, Cambridge: University of Cambridge; 1992, p.40-1 (Abstr).

29. SCHRYVER,H.F.,HINTZ,H.F.;CRAIG,P.H.: Phosphorus metabolism in ponies fed varying levels of phosphorus. Journal of Nutrition , v.101, p1257-64, 1971.

30. SCHRYVER,H.F.; HINTZ, H.F.: Recent developments in equine nutrition. Animal Nutrition and Health, v.4, p6-10, 1975

31. SCHRYVER,H.F.; HINTZ,H.F. AND LOWE, J.E.: Calcium and phosphorus nutrition of the horse. Cornell Veterinary, v.64, p491-515, 1974

32. TRAVER,D.S., et al: Renal metabolism of endogenous substances in the horse : Volumetric vs Clearance ratio methods. Journal of Equine Medicine Surgery v.1,n.11, p378-82, 1977.

33. WALTHALL,J.C.; MCKENZIE,R.A.: Osteodystrofia fibrosa in horses at pasture in Queensland: field and laboratory observations. Australian Veterinary Journal, v.52, p1116,1976

34. WARREN,J.A.: The effects of exercise and age on PTH concentrations in weanling horses. 1991, MS Thesis, Dept of An.Sci and Ind, Kansas State Univ, Manhatan.

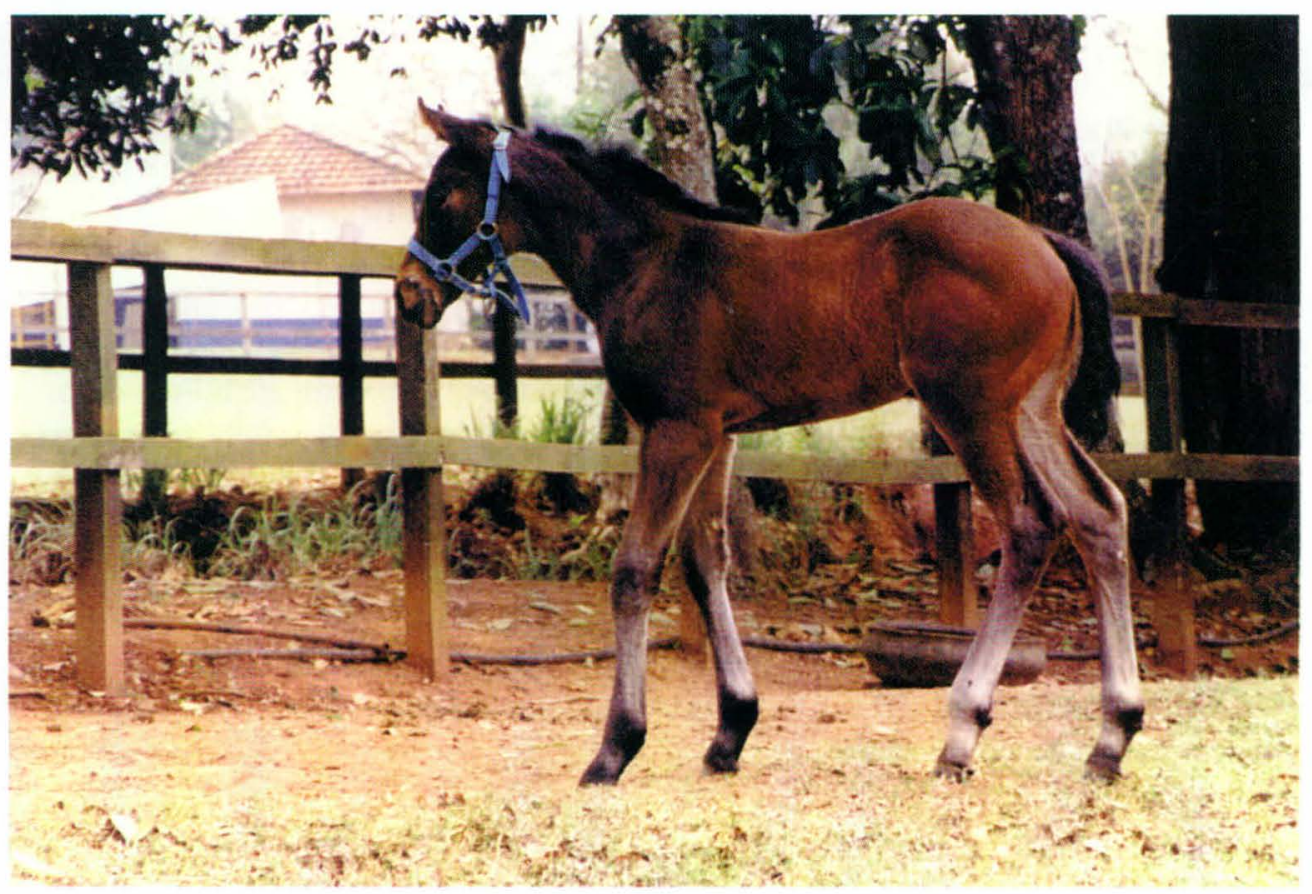

